\title{
GMT Integral-Field Spectrograph (GMTIFS) conceptual design
}

\author{
Peter J. McGregor ${ }^{\mathrm{a}}$, G. J. Bloxham ${ }^{\mathrm{a}}$, R. Boz ${ }^{\mathrm{a}}$, J. Davies ${ }^{\mathrm{a}}$, M. Doolan ${ }^{\mathrm{a}}$, M. Ellis ${ }^{\mathrm{a}}$, J. Hart ${ }^{\mathrm{a}}$, D. J. Jones ${ }^{\mathrm{b}}$,

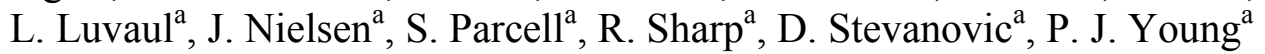 \\ ${ }^{a}$ Research School of Astronomy and Astrophysics, College of Physical and Mathematical Sciences, \\ The Australian National University, Cotter Road, Weston, ACT, 2611, Australia \\ ${ }^{\mathrm{b}}$ Prime Optics, 17 Crescent Rd., Eumundi, Queensland 4562, Australia
}

\begin{abstract}
The Giant Magellan Telescope (GMT) Integral-Field Spectrograph (GMTIFS) ${ }^{c}$ is one of six potential first-light instruments for the 25m-diameter Giant Magellan Telescope. The Australian National University has completed a Conceptual Design Study for GMTIFS. The science cases for GMTIFS are summarized, and the instrument capabilities and design challenges are described. GMTIFS will be the work-horse adaptive-optics instrument for GMT. It contains an integral-field spectrograph (IFS) and Imager accessing the science field, and an On-Instrument Wave-Front Sensor (OIWFS) that patrols the 90 arcsec radius guide field. GMTIFS will address a wide range of science from epoch of reionization studies to forming galaxies at high redshifts and star and planet formation in our Galaxy. It will fully exploit the Laser Tomography Adaptive Optics (LTAO) system on the telescope. The tight image quality and positioning stability requirements that this imposes drive the design complexity. Some cryogenic mechanisms in the IFS must set to $\sim 1 \mu \mathrm{m}$ precision. The Beam-Steering mechanism in the OIWFS must set to milli-arcsecond precision over the guide field, corresponding to $\sim 1 \mu \mathrm{m}$ precision in the $\mathrm{f} / 8$ focal plane. Differential atmospheric dispersion must also be corrected to milli-arcsecond precision. Conceptual design solutions addressing these and other issues are presented and discussed.
\end{abstract}

Keywords: GMT, near-infrared, integral-field spectrograph

\section{INTRODUCTION}

The Giant Magellan Telescope will be a revolutionary scientific facility. Its great light-gathering power will allow the high-redshift Universe to be probed in exquisite detail. Its large aperture will permit diffraction-limited adaptive-optics observations at unprecedented angular resolution; 3.8 times better than the James Webb Space Telescope (JWST) at the same wavelength.

GMTIFS will exploit both of these GMT advantages. Its combination of an adaptive-optics-corrected integral-field spectrograph with an adaptive-optics imager matched to the corrected field of the GMT LTAO system will make GMTIFS the workhorse adaptive-optics instrument for the GMT community.

The key features of the GMTIFS IFS are its high-angular-resolution integral-field unit, its range of four image scales, and its moderate spectral resolution in the 0.9-2.5 $\mu \mathrm{m}$ wavelength range. This combination maximizes the information gathering potential. The type of observations for which the GMTIFS IFS will excel require high-spectral-resolution data of spatially complex regions having high surface brightness in either a spatial or spectral sense. Examples of these two extremes are spatially compact, continuum sources (e.g., resolved stars) and spatially extended, narrow emission-line regions (e.g., ionized gas). These themes feature strongly in the GMTIFS IFS science drivers.

The key features of the GMTIFS Imager are its 5 mas pixels and $20.4 \times 20.4 \operatorname{arcsec}$ field of view. This pixel scale Nyquist samples the LTAO-corrected point-spread function (PSF) of GMT at the shortest operating wavelength of $\sim 1.0 \mu \mathrm{m}$ and slightly over-samples the PSF at longer wavelengths, as is required for high photometric precision. An important role of the Imager will be in providing complementary photometric data on the stellar components of objects at the same angular resolution as their gaseous components are studied with the GMTIFS IFS. The Imager will also act as an efficient acquisition camera for the IFS at its finest image scales and smallest fields of view.

\footnotetext{
${ }^{\mathrm{c}}$ http://www.mso.anu.edu.au/gmtifs
}

Ground-based and Airborne Instrumentation for Astronomy IV, edited by lan S. McLean, Suzanne K. Ramsay, Hideki Takami, Proc. of SPIE Vol. 8446, 84461I · @ 2012 SPIE · CCC code: 0277-786X/12/\$18 · doi: 10.1117/12.925259 
High sky coverage will be essential for adaptive-optics instruments on $30 \mathrm{~m}$ class telescopes in the 2020s. The GMT adaptive secondary mirror allows a large guide field to be passed efficiently to adaptive-optics instruments, giving GMT a potential sky coverage advantage over other telescopes. GMTIFS capitalizes on this advantage by accepting a 180 arcsec diameter guide field.

The science potential of GMTIFS is intimately tied to the requirements of the GMT LTAO system. To exploit the full diffraction-limited performance of GMT, the LTAO system requires a tip-tilt guide star brighter than $K \sim 17$ mag to be located within $\sim 30 \mathrm{arcsec}$ of the science object. Strehl ratios of $\sim 0.14,0.35$, and 0.55 are then expected at $J, H$, and $K$, respectively, in good seeing conditions. The corresponding diffraction-limited angular resolution will be $\sim 13$ mas at $J, \sim$ 16 mas at $H$, and $\sim 22$ mas at $K$. Many GMTIFS IFS and Imager observations will be performed in this mode.

Other GMTIFS IFS observations will exploit the light collecting power of GMT using coarser spatial sampling. These observations do not require such high image stability because the image scale used does not fully sample the diffractionlimited PSF. Then ensquared energy is a better adaptive-optics performance metric than Strehl ratio. These IFS observations with coarse spaxels ${ }^{\mathrm{d}}$ will be possible using natural guide stars located anywhere within the 180 arcsec diameter guide field.

GMTIFS will achieve $>90 \%$ sky coverage for coarse-spaxel ensquared-energy observations and $\sim 30 \%$ sky coverage for diffraction-limited observations using open-loop correction of a single off-axis guide star.

Like all adaptive-optics instruments, the scientific productivity of GMTIFS will benefit from queue-scheduling of GMT to utilize the best seeing conditions.

\section{SCIENCE DRIVERS}

The era of 8-10 m telescopes saw revolutionary advances in adaptive optics and integral-field spectroscopy that placed high angular resolution astronomy at the scientific frontier. Laser Guide Star (LGS) adaptive-optics systems made high angular resolution science on ground-based telescopes generally accessible over a broad range of fields. Coupling these adaptive-optics systems to near-infrared integral-field spectrographs, such as NIFS on Gemini, OSIRIS on Keck, and SINFONI on the VLT, enabled adaptive-optics science to transition from descriptive morphological studies to quantitative studies of kinematics, excitation, and chemistry that probe physical mechanisms occurring in a wide range of astrophysical objects. These are now essential capabilities for the next generation of Extremely Large Telescopes.

GMTIFS will address many of the key science drivers for the GMT telescope spanning the full range of cosmic history:

- Observations of the near-infrared counterparts of Gamma-Ray Bursts will probe the structure of the intergalactic medium at the epoch of reionization beyond $z \sim 7$ using the high spectral resolution of the GMTIFS IFS.

- The mass assembly of galaxies over cosmic time will be studied using kinematic measurements of Ly $\alpha$ and $\mathrm{H} \alpha$ emission that exploit the high sensitivity of the GMTIFS IFS. The broad wavelength coverage of the IFS will be used to study the associated chemical evolution of these galaxies via spatially-resolved measurements of their restframe-optical emission-line ratios. The high angular resolution of the GMTIFS Imager will be used to probe the build-up of the stellar components of these galaxies via broad-band near-infrared imaging. In these ways, GMTIFS will provide essential follow-up observations for the large samples of high-redshift galaxies that will be identified at lower angular and spectral resolution with JWST.

- The GMTIFS IFS will probe the most massive nuclear black holes in the Universe via high-angular-resolution measurements of stellar kinematics in some objects and Keplerian motions of circumnuclear gas in other objects. These observations will clarify the intimate relationship between nuclear black-hole mass and host galaxy stellar properties at the highest black-hole masses, and elucidate the upper black-hole mass limit and its evolution over time.

- The GMTIFS IFS will also probe the least massive nuclear black holes in nearby galaxies and investigate the duality of low-mass black holes and nuclear star clusters as alternative end-products of bulge formation in lower mass galaxies.

\footnotetext{
d In integral-field spectroscopy, a "spaxel" is a spatial pixel while a "pixel" is a spectral pixel.
} 
- The high angular resolution of the GMTIFS Imager will be used to resolve individual stars in galaxies beyond the Local Group, providing direct insights into the star formation and chemical histories of complex stellar systems in different environments.

- The high angular resolution and high spectral resolution of the GMTIFS IFS will combine to probe the jet outflows and planet-forming circumstellar disks associated with nearby forming stars in unprecedented detail. This will reveal the internal structure of the outflows and constrain the launch physics of their jets. IFS measurements of nearinfrared emission lines originating at the disk surface will complement $\mathrm{mm}$-wave probes of the disk interior exploited with ALMA.

- The high angular resolution of the GMTIFS IFS will allow spectroscopic studies of outer exosolar planets identified in near-term Extreme Adaptive Optics surveys on 8-m telescopes.

\section{INSTRUMENT DESCRIPTION}

\subsection{Instrument Parameters}

The GMTIFS instrument consists of a diffraction-limited science IFS, a diffraction-limited science Imager, an OIWFS patrolling the 90 arcsec radius guide field, as well as an instrument calibration system. The unfolded optical system is shown in Figure 1. The instrument subsystems are shown schematically in Figure 2. The optical adaptive-optics wavefront sensors (WFSs) that mount on the outside of the GMTIFS cryostat are essential for the operation of the instrument, but are not formally part of it. The top-level parameters of the main GMTIFS subsystems are summarized in Table 1.

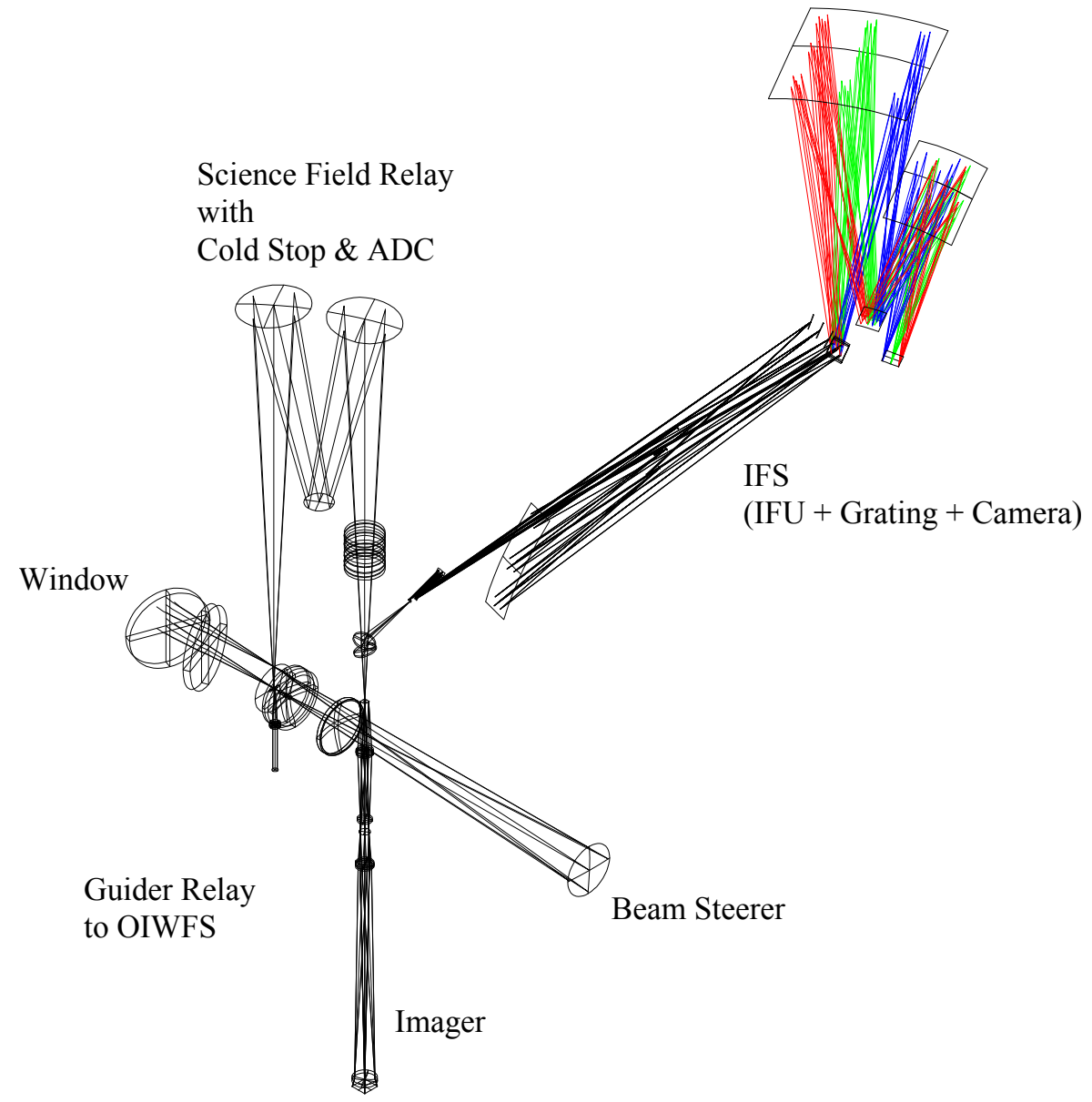

Figure 1: Isometric view of the unfolded optical systems that are housed within the GMTIFS cryostat. Light enters the instrument through the window at middle-left and passes either to the IFS, Imager, or the OIWFS. 


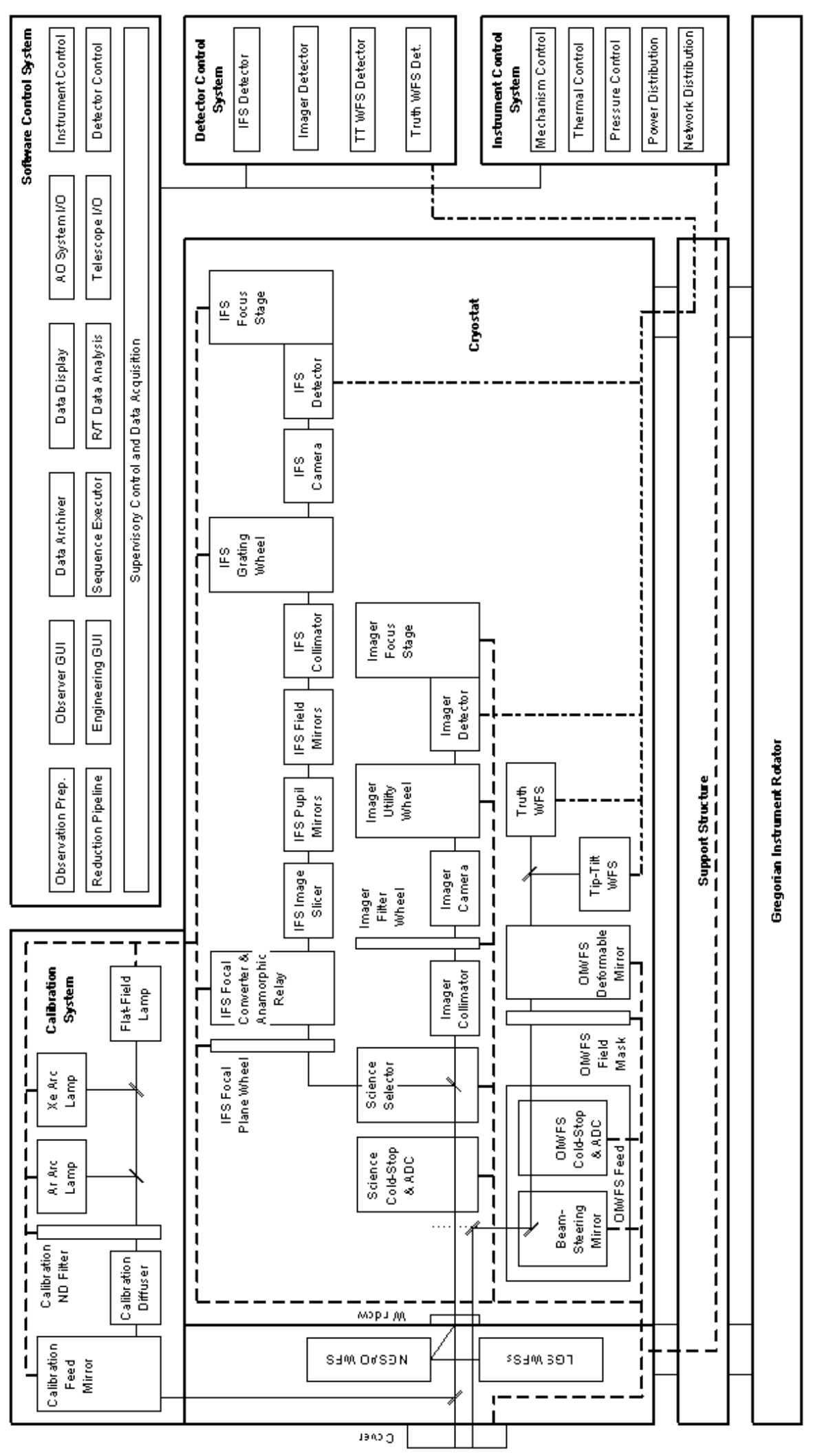

Figure 2: GMTIFS subsystem schematic. 
Table 1: Top-Level GMTIFS Parameters

\begin{tabular}{|c|c|}
\hline Integral-Field Spectrograph & \\
\hline IFS feed: & Dichroic mirror reflection \\
\hline Wavelength range: & $0.9-2.5 \mu \mathrm{m}$ \\
\hline Field geometry: & 45 slitlets $\times 88$ spaxels per slitlet \\
\hline Spaxel size: & $50,25,12,6$ mas, square on sky \\
\hline Field of view: & $4.40 \times 2.25,2.20 \times 1.125,1.056 \times 0.54,0.528 \times 0.27$ arcsec \\
\hline Field location: & $7.29,3.65,1.83,0.91$ arcsec with respect to the optical axis \\
\hline Spectral resolving power: & $\begin{array}{l}5,000(m Z J, m J H, m H K) \\
10,000(h Z, h J, h H, h K)\end{array}$ \\
\hline Spectral coverage: & $\begin{array}{l}0.89-1.35 \mu \mathrm{m}(m Z J) \\
1.19-1.80 \mu \mathrm{m}(m J H) \\
1.64-2.49 \mu \mathrm{m}(m H K) \\
0.92-1.13 \mu \mathrm{m}(h Z) \\
1.10-1.35 \mu \mathrm{m}(h J) \\
1.47-1.80 \mu \mathrm{m}(h H) \\
2.04-2.51 \mu \mathrm{m}(h K)\end{array}$ \\
\hline Focal-plane occulting masks & $25,50,100,200$ mas diameter circular \\
\hline Target acquisition: & Imaging mode or collapsed IFS data cube \\
\hline Detector: & Teledyne 4096×4096 pixel HgCdTe HAWAII-4RG-15 \\
\hline Guide mode: & Tip-Tilt Guider or Imager On-Detector Guide Window \\
\hline \multicolumn{2}{|l|}{ Imager } \\
\hline Imager feed: & Direct or dichroic transmission \\
\hline Wavelength range: & $0.9-2.5 \mu \mathrm{m}$ \\
\hline Pixel size: & 5 mas \\
\hline Field of view: & $20.4 \times 20.4$ arcsec square \\
\hline Filters: & $J H K+$ intermediate bands + narrow bands \\
\hline Target acquisition: & Imaging mode \\
\hline Detector: & Teledyne 4096×4096 pixel HgCdTe HAWAII-4RG-15 \\
\hline Guide mode: & Tip-Tilt Guider or Imager On-Detector Guide Window \\
\hline Cold pupil stop alignment: & Pupil-viewing optics \\
\hline Non-common path wave front: & Dual defocus lenses \\
\hline \multicolumn{2}{|c|}{ On-Instrument Wave-Front Sensor } \\
\hline Wavelength range: & $0.9-2.5 \mu \mathrm{m}$ \\
\hline Patrol field: & 180 arcsec diameter circular, excluding Imager field \\
\hline Guide star selection & Beam-steering mirror at pupil image \\
\hline \multicolumn{2}{|l|}{ Calibration } \\
\hline IFS dark exposures: & IFS optical path blocked \\
\hline Imager dark exposures: & Imager optical path blocked \\
\hline Wavelength calibration: & $\mathrm{Ar}+\mathrm{Xe}$ arc lamps \\
\hline Flat field calibration: & Incandescent lamp \\
\hline Intensity attenuation: & Neutral density filters matched to all science scales \\
\hline Illuminated field of view & $20.4 \times 20.4$ arcsec Imager field \\
\hline
\end{tabular}




\subsection{Adaptive-Optics Systems}

GMTIFS will be located on the upper level of the Gregorian Instrument Rotator (Figure 3). It will be fed efficiently by the $\mathrm{f} / 8$ beam from the adaptive secondary mirror via only a single reflection at the telescope tertiary mirror. The tilted GMTIFS cryostat window reflects light shortward of $0.9 \mu \mathrm{m}$ to external optical adaptive-optics wave-front sensors while transmitting near-infrared light to the instrument.

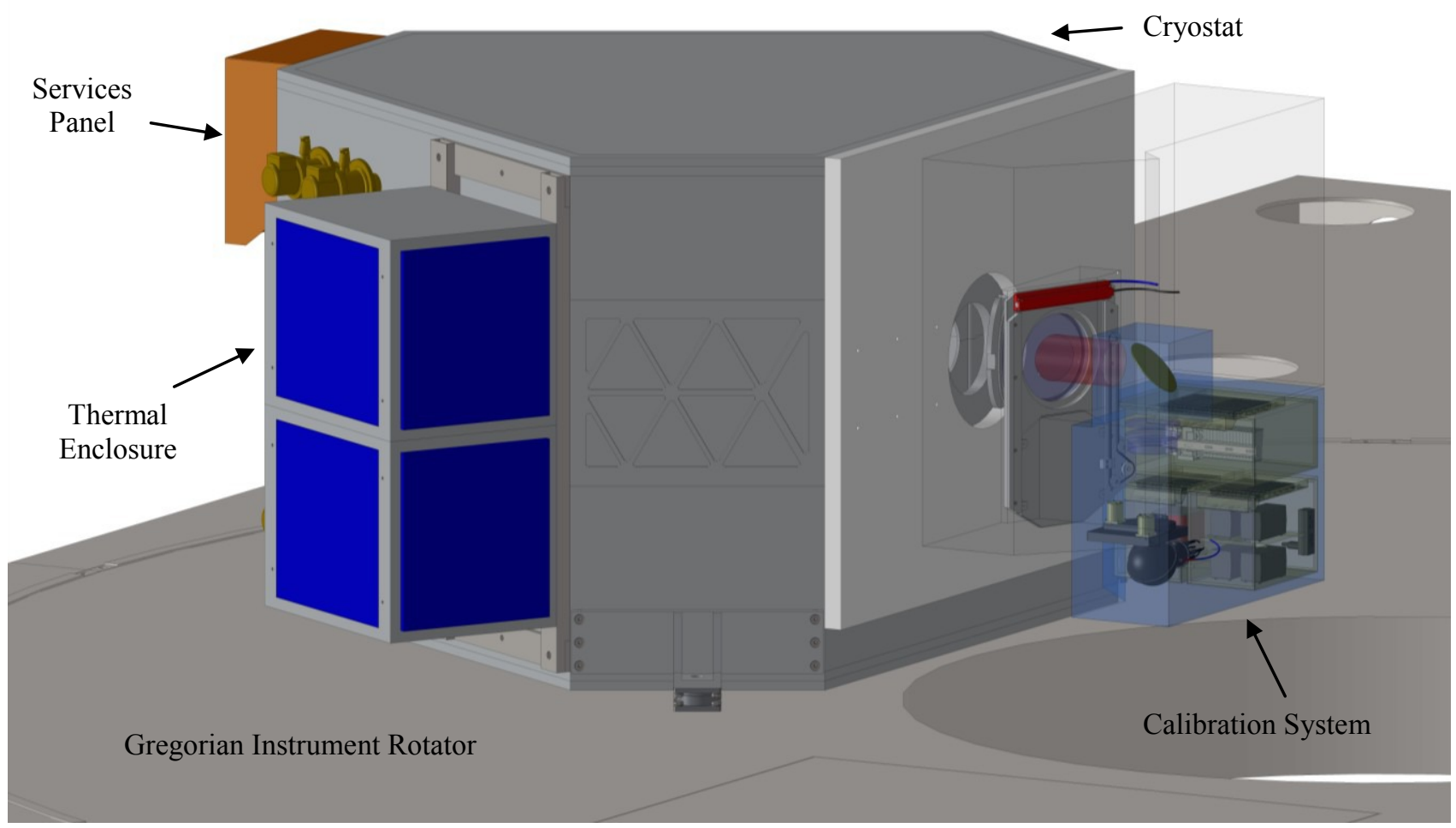

Figure 3: GMTIFS cryostat on the GMT Gregorian Instrument Rotator.

GMTIFS will be used with the GMT Natural Guide Star Adaptive Optics (NGSAO) system where possible. This is the preferred adaptive-optics mode for GMTIFS when a suitably bright guide star is available at the field center. Use of the NGSAO mode avoids the need to clear calibration targets with Space Command prior to laser propagation. It also delivers better image quality than the LTAO system by correcting atmospheric piston terms that cannot be corrected by the LTAO system.

While the NGSAO system will provide a higher level of adaptive-optics correction than the LTAO system, locating the fundamental position reference (i.e., the pyramid Wave-Front Sensor (WFS)) on the outside of the cryostat means that differential motions between the cryostat interior and exterior may be problematic. This flexure can be monitored using the GMTIFS Imager On-Detector Guide Window (ODGW) or the near-infrared tip-tilt WFS in the GMTIFS OIWFS.

GMTIFS will most often be used behind GMT's LTAO system, and so will operate in the 1-2.5 $\mu$ m wavelength region where this system performs well. The LTAO system uses GMT's adaptive secondary mirror, six LGSs, and at least one Natural Guide Star (NGS) to deliver a diffraction-limited field of $\sim 30 \operatorname{arcsec}$ diameter to the science instrument. The angular resolution within this field ranges from $\sim 10$ mas at a wavelength of $1.0 \mu \mathrm{m}$ to $\sim 25$ mas at $2.5 \mu \mathrm{m}$.

High-order wave-front monitoring is provided by the six LGS WFSs. These cannot measure science image position or focus, so low-order aberrations are measured by the OIWFS using the near-infrared, adaptive-optics-corrected image of an off-axis natural guide star. The fast near-infrared tip-tilt WFS in the OIWFS monitors tip-tilt motions. The slow nearinfrared Truth WFS in the OIWFS performs three functions: Firstly, it monitors science focus independent of variations in the distance to the atmospheric Na layer as the telescope tracks to different zenith distances. Secondly, the nearinfrared Truth WFS monitors the time-averaged image quality so that low-order biases can be corrected in the LGS WFS 
spot centroids due to the changing profile of $\mathrm{Na}$ atoms through the atmospheric Na layer. Thirdly, the near-infrared Truth WFS monitors wave-front piston differences between the seven segments of the GMT telescope.

The near-infrared image of the off-axis guide star presented to the GMTIFS OIWFS will benefit from some level of adaptive-optics correction via the adaptive secondary mirror. This will range from full diffraction-limited correction near the optical axis to partial correction at the extremity of the 90 arcsec radius guide field. A second deformable mirror in the GMTIFS OIWFS provides additional open-loop adaptive-optics correction for off-axis guide stars based on the tomographic wave-front reconstruction from the LGS WFSs. This ensures that the image of the OIWFS guide star is always near diffraction-limited.

Correction of tip-tilt motions is expected to be most demanding for the LTAO system. This includes tip-tilt motions due to the atmosphere, windshake, and vibrations. Atmospheric tip-tilt is best monitored using a star close to the science target (due to angular anisoplanatism), but windshake and vibrations can be monitored anywhere in the guide field. It is therefore preferable to measure atmospheric tip-tilt near the science target using the Imager ODGW when a suitable star is available within the science field. During IFS observations, tip-tilt motions can be monitored with the Imager ODGW in out-of-band light from suitable science targets using a dichroic Science Selector element that reflects primary science wavelengths to the IFS while passing complementary wavelengths to the Imager. Often it will be most appropriate to guide on the science target itself, or some compact component of it. Tip-tilt motions due to windshake and vibrations can still be measured using a more distant OIWFS guide star feeding the near-infrared tip-tilt WFS, with the two tip-tilt measurements being combined tomographically.

\subsection{GMTIFS Fore-Optics}

The GMTIFS cryostat accepts the 3.0 arcmin diameter field passed to it from the GMT adaptive secondary mirror. This field enters GMTIFS through a $300 \mathrm{~mm}$ diameter cryostat window. The cryostat window has a dichroic coating and is tilted to reflect optical light to the NGSAO and LGS WFSs. Aberrations (mainly astigmatism) due to this tilted window must be corrected in the transmitted beam. To achieve this, the window element is wedged and a second tilted and wedged corrector plate is mounted immediately behind the cryostat window (Figure 4). The two plates have the same wedge angle, but are oriented in opposite directions. The plates are also tilted in opposite directions, but with a slight difference in angle as required to eliminate angular deviation of the chief ray. There is then almost no aberration in the pupil image except for anamorphic distortion. Importantly, there is also no significant tilt of the focal plane with respect to the optical axis because the combined optical thickness is almost independent of field angle. Longitudinal color is the only significant field aberration introduced by the corrected window. This is compensated in the science beam by the atmospheric dispersion corrector.

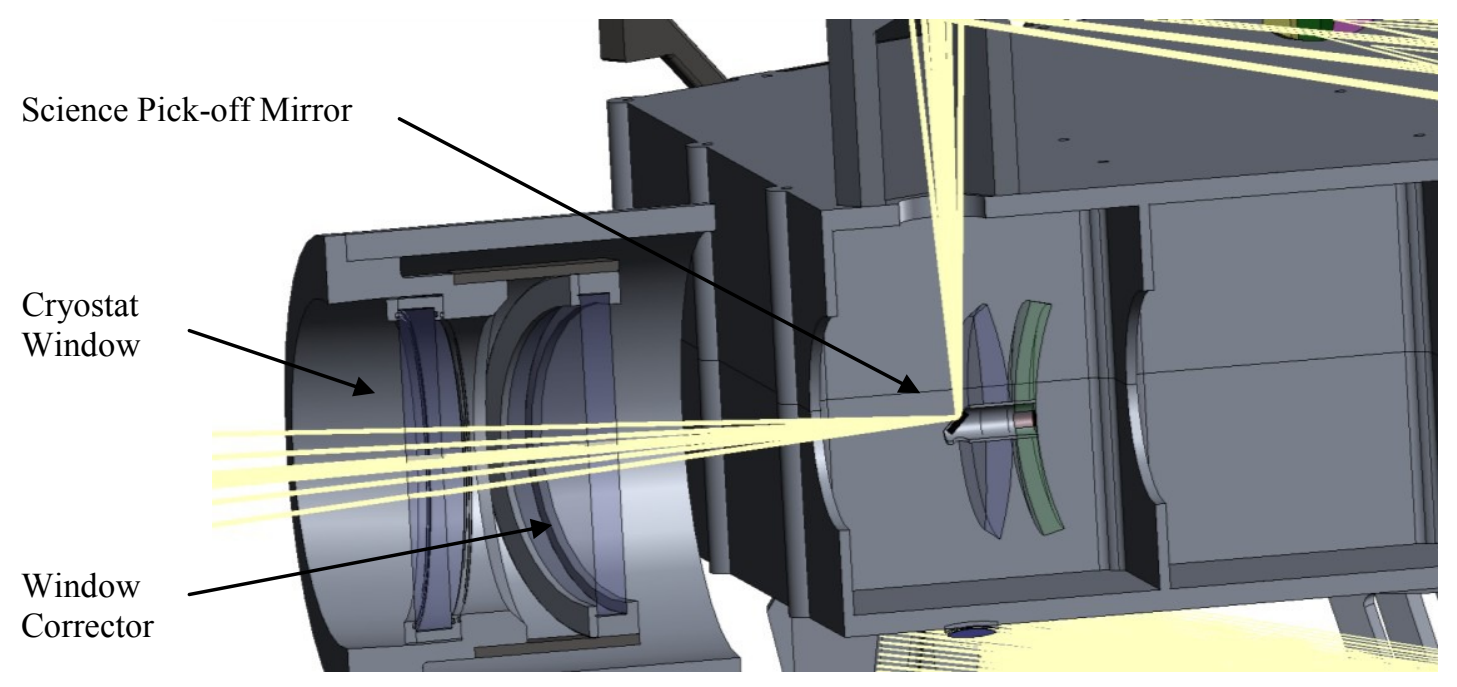

Figure 4: GMTIFS window, window corrector, and science field feed. 
The $20.4 \times 20.4$ arcsec science field is then reflected upwards within the cryostat (Figure 4) to reimaging science foreoptics in the form of an Offner relay (Figure 5) that performs three tasks; it relays the science focal plane to a convenient location, it forms an image of the GMT exit pupil at a rotating cold stop baffle, and it provides the path length needed to correct atmospheric dispersion in the science beam.

The first mirror of the Offner relay forms a pupil image on the second mirror. Astigmatism in the pupil image is corrected by figuring the science pick-off mirror as a weak toric. The science cold stop baffle has the center-hexagonal shape of the GMT primary mirror. The pupil image rotates about the optical axis as the Gregorian Instrument Rotator derotates the field when tracking a target. Consequently, the cold stop mask must be actively driven in angle to maintain its alignment with the pupil image.

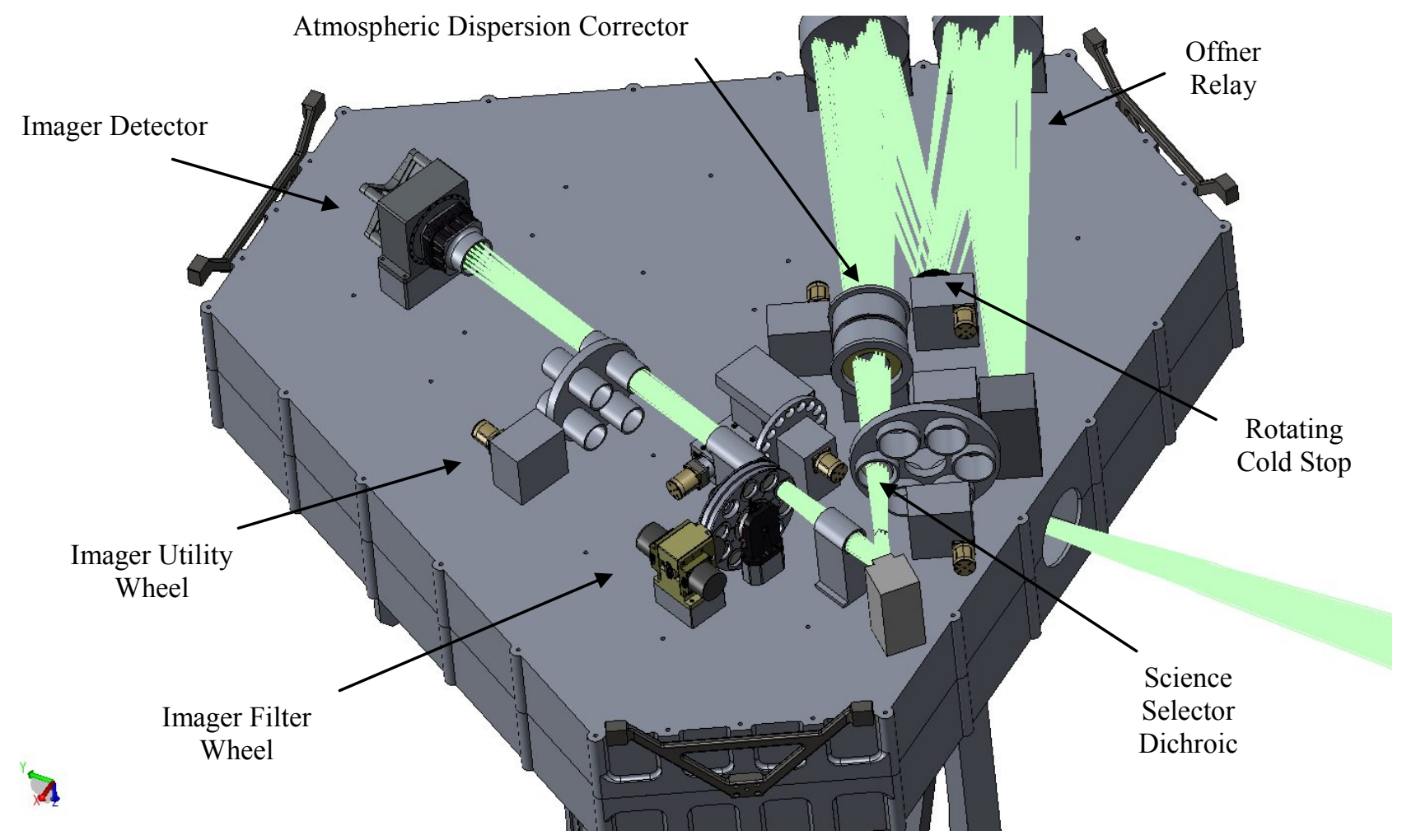

Figure 5: GMTIFS Fore-Optics and Imager.

The unprecedented high angular resolution attainable with LTAO on GMT places strong constraints on image quality. Principle among these is the need to accurately correct differential atmospheric dispersion over the science wavelength band. The GMTIFS fore-optics contains a multi-component atmospheric dispersion corrector for this purpose. The conceptual design uses two sets of four prism plates in a converging beam. The atmospheric dispersion corrector is placed in the long output beam of the science relay. The design is made complex by the lack of beam collimation, which requires the use of curved surfaces.

The science ADC must correct atmospheric dispersion across any one science observation band to better than $10 \%$ of the diffraction-limited full width at half maximum (FWHM) at the science wavelength. It must also correct atmospheric dispersion in the guiding pass band when using the Imager ODGW for tip-tilt monitoring to an accuracy that allows the guide star image centroid to be determined to better than $10 \%$ of the diffraction-limited FWHM at the science wavelength.

A Science Selector mechanism (Figure 5) then directs the science beam either to the IFS or to the Imager. IFS observations use a dichroic mirror that reflects the science wavelength band to the IFS and transmits out-of-band science 
light to the Imager. This allows the Imager to view the IFS science field (in out-of-band light). Aberrations in the transmitted beam are corrected with a second tilted corrector plate, similar to the cryostat window corrector. A flat nontilted fused silica plate will be used to direct all of the near-infrared light to the Imager for Imager science observations.

\subsection{Integral-Field Spectrograph}

The GMTIFS IFS provides a range of four image scales and corresponding rectangular fields of view (Table 1). The coarsest scale samples faint targets with $\sim 50$ mas sampling. The fine scales sample the diffraction FWHM over a range of wavelengths. The rectangular field of view with an aspect ratio of 2:1 permits in-field nodding of compact science targets for accurate sky-subtraction with optimal efficiency. The use of square spaxels on the sky simplifies data analysis. Two moderate spectral resolving powers will be provided. The velocity resolution of $\sim 60 \mathrm{~km} \mathrm{~s}^{-1}$ achieved at $R$ $\sim 5,000$ samples dynamical motions in large galaxies and allows access to significant low-background wavelength regions between strong airglow emission lines. The higher resolution $R \sim 10,000$ gratings produce velocity resolutions of $\sim 30 \mathrm{~km} \mathrm{~s}^{-1}$ that probe stellar kinematics in the vicinities of lower mass $\left(10^{4}-10^{6} \mathrm{M}_{\text {Sun }}\right)$ black holes and nuclear star clusters.

The dichroic mirror in the Science Selector that feeds the IFS will provide sufficient spectral order blocking.

The IFS beam first passes to a Focal Plane Wheel (Figure 6). This wheel contains the rectangular IFS field masks, the spatial calibration masks (Ronchi gratings ${ }^{\mathrm{e}}$ ), occulting disks, and a blocked position for recording dark exposures. The wheel contents should be positioned laterally in the optical beam with a repeatability of $<10 \%$ of the diffraction FWHM at the shortest operating wavelength of $0.89 \mu \mathrm{m}$. This equates to $\sim 0.9 \mu \mathrm{m}$ in the $\mathrm{f} / 8$ focal plane.

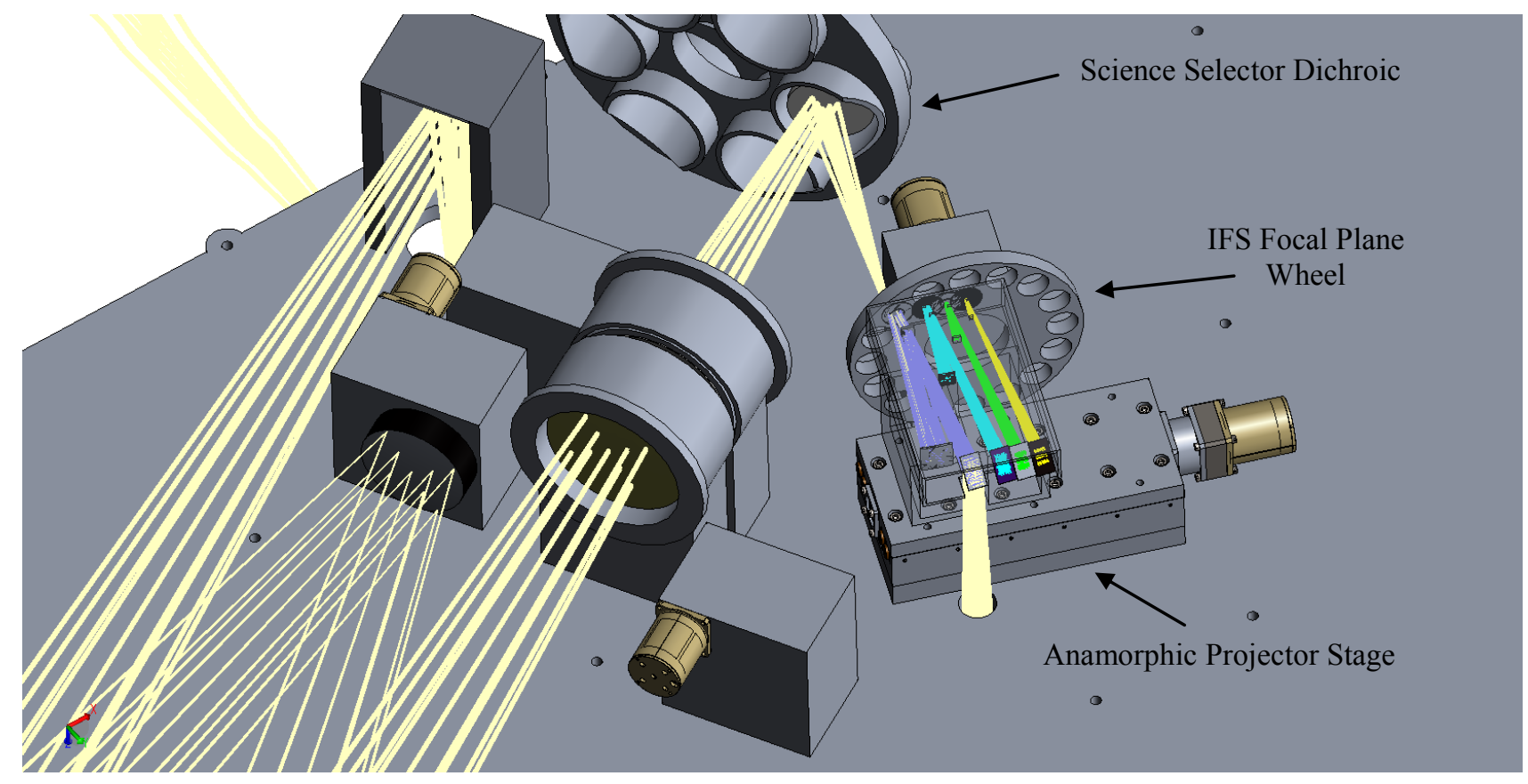

Figure 6: GMTIFS Science Selector mechanism, Focal Plane Wheel, and Anamorphic Projector systems.

The IFS Focal Plane Wheel is followed immediately by matched anamorphic projectors (Figure 6). These two-mirror systems project input fields of different extents to a fixed field size at the image slicer in the integral-field spectrograph. At the same time, they maintain a fixed pupil position that is conjugate to the IFS pupil mirrors and gratings, and they introduce a 2:1 anamorphic magnification that results in square spaxels projected back to the sky.

\footnotetext{
${ }^{\mathrm{e}}$ Ronchi gratings are coarse transmission gratings oriented perpendicular to the IFS slitlets to simulate multiple evenlyspaced input sources illuminating each slitlet.
} 
GMTIFS extends the NIFS concentric IFU design ${ }^{1}$ by using a selection of four of these anamorphic projectors to provide four different image scales (Table 1) at the image-slicer mirrors. While the pupil position is the same for each image scale, the pupil diameter changes so that the pupil mirrors and gratings must be sized to accommodate the coarsest pixel scale. This ensures that these elements are sufficiently over-sized to capture most of the light that is diffracted by the image-slicer mirrors to extreme angles when using the finer image scales.

The four anamorphic projectors are geometrically similar, differing only in that their size is proportional to the field size. They are comprised of two toric mirrors that project the small input field to the image slicer at large magnification. The mirrors are arranged so that the virtual output pupil lies on the same plane as the input field. The scaling made between projectors occurs about the center of the elliptical output pupil so that downstream images of the pupil are concentric and can share the same optics. The various input fields are then offset from this pupil center in proportion to their size.

The anamorphic projectors must be positioned in the IFS beam to the same $\sim 1 \mu \mathrm{m}$ accuracy as the IFS Focal Plane Wheel. In this case, a precision linear stage is proposed (Figure 7). The mechanism uses rolling-element bearings for both the guiding and actuating systems. Linear translation is based on a 500 step Phytron VSS UHVC cryogenic stepper motor that provides input to a 2.5:1 planetary reduction stage. The reduction stage directly drives a recirculating roller screw with $1.0 \mathrm{~mm}$ pitch through a torsionally-stiff bellows-type coupling. The recirculating roller screw features two nuts with soft preloading applied through a series of bearing preload washers. The range of travel is $50 \mathrm{~mm}$ with a resolution of $0.8 \mu \mathrm{m}$ per motor step. A full traverse of the carriage takes 31 seconds. A diaphragm flexure is proposed for the lead screw floating bearing mount in order to control thermal load transfer to bearing interfaces. This allows conformance in the axial direction while remaining laterally stiff.

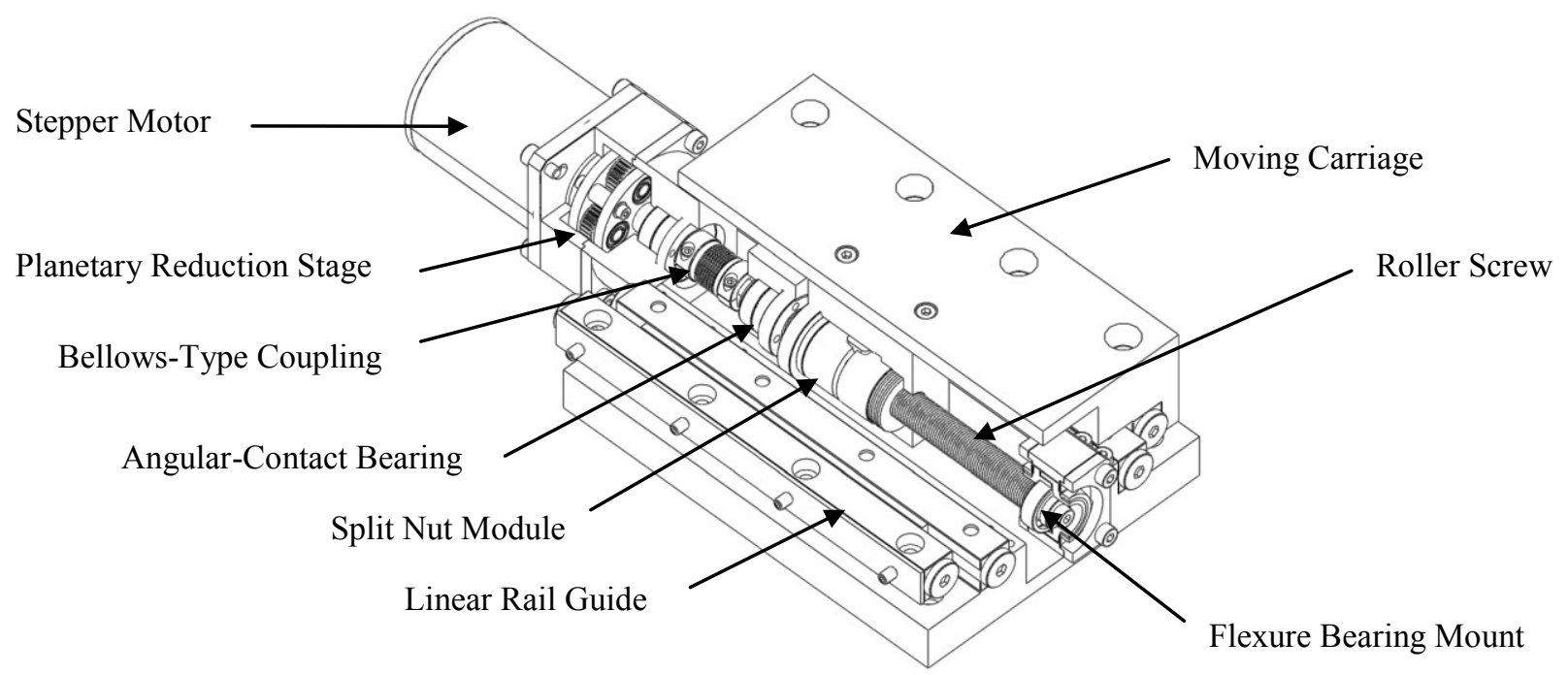

Figure 7: Cutaway view of the GMTIFS anamorphic projector stage.

The IFS beam exiting the anamorphic projector is then folded down (Figure 6) and passes through the central optical bench to the IFS proper.

The design of the GMTIFS IFS is based on the strong heritage of integral-field spectrographs that have been designed and constructed at the Australian National University (ANU). Like the Near-infrared Integral-Field Spectrograph ${ }^{2}$ (NIFS) that was built by the ANU for the Gemini North telescope, the GMTIFS IFS (Figure 8) will use the concentric integral-field unit (IFU) principle and diamond-machined metal optics to reformat the input field into a long slit.

The concentric integral-field unit uses the imager-slicer concept. The IFU channels are fanned about a single axis and mirror arrays are used that are concentric with that axis. Thus all IFU channels experience similar low optical aberrations in much the same way that the spherical mirrors of a Schmidt telescope have no preferred axis. This minimizes image aberrations to achieve diffraction-limited performance over large fields. 


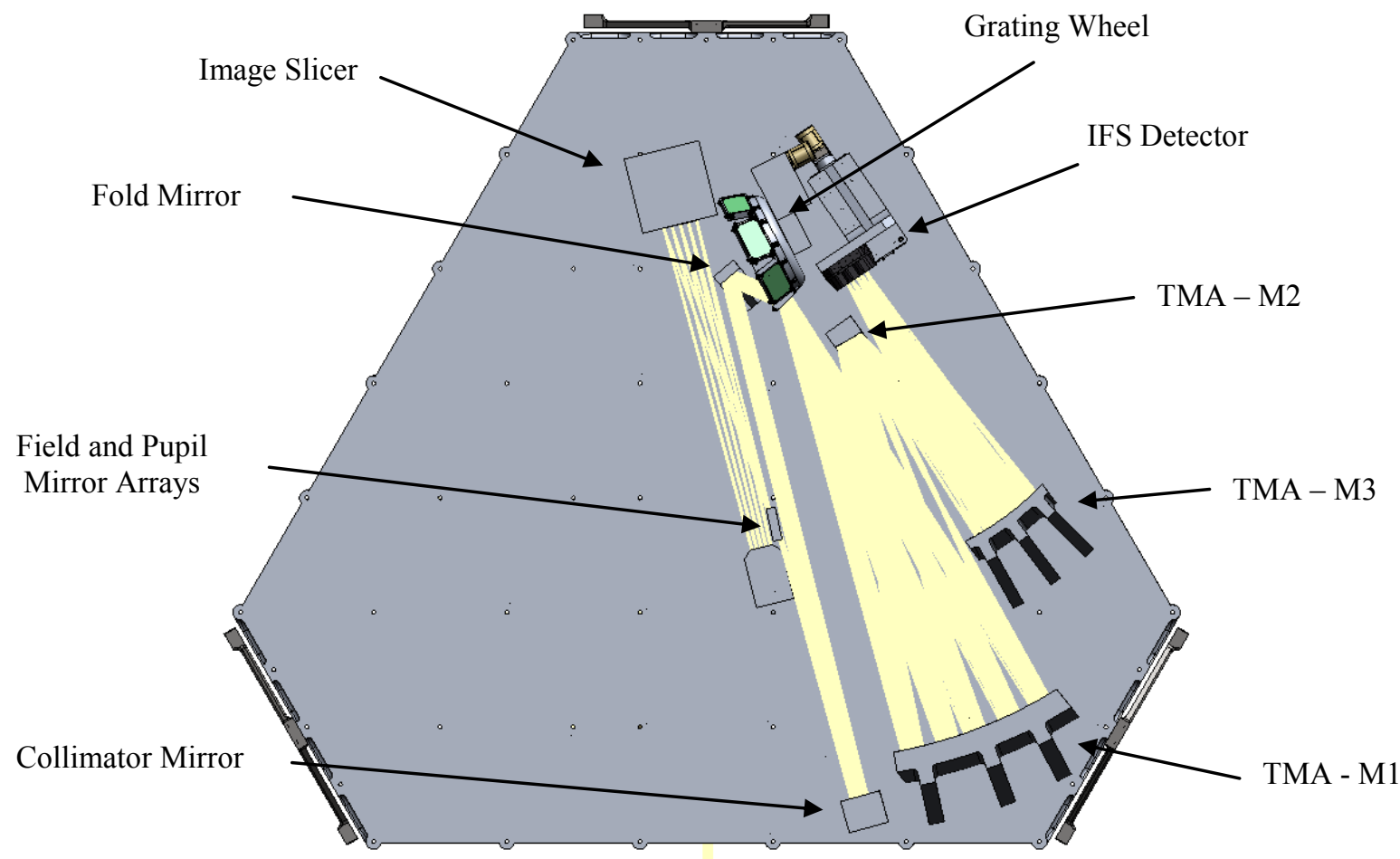

Figure 8: GMTIFS IFS mounted on the lower side of the optical bench.

The beam from the anamorphic projector is imaged onto the slicer mirrors at an appropriate scale. The image-slicer mirrors are concave and fanned so that they reimage the telescope exit pupil downstream on an array of pupil mirrors. These pupil mirrors are also powered so that they reimage the image-slicer field onto an array of field mirrors in the form of a long "staircase" slit image. The field mirrors are also powered so that they direct the beams from each position along any one slitlet towards the grating pupil. Both the pupil and field mirror arrays are arranged along arcs about the fanning axis of the image slicer so each IFU channel is optically similar, and their images are not degraded by the increasing offaxis angles that would otherwise result.

The beam from the field mirrors passes to the spherical spectrograph collimator mirror, which is also concentric with the image-slicer fanning axis. The collimator reimages each IFU channel to a common pupil at the spectrograph grating.

Diffraction effects at the image-slicer slitlets widen the beam in the dispersion direction. However, the over-sized optics required for the coarsest spatial scales succeed in capturing most of this diffracted light.

Diamond-machined metal mirrors permit mono-metallic construction of the IFU. Their use in monolithic multi-surface mirror arrays greatly simplifies optical alignment because the intrinsic accuracy of the diamond-turning machine is transferred directly to the optic. Furthermore, recent developments in diamond-turning technologies and materials have resulted in significantly smoother optics than used in NIFS, with correspondingly much lower scattered-light levels.

As with the Wide-Field Spectrograph (WiFeS) optical integral-field spectrograph ${ }^{3}$ built at the ANU, the GMTIFS IFS will use a suite of Volume Phase Holographic (VPH) transmission gratings as the dispersing elements. These efficient gratings offer high throughput and can be manufactured with near-uniform response over each of the required wavelength bands.

The VPH gratings are arranged to work in a Littrow configuration. By operating at Littrow, the gratings do not introduce angle-dependent anamorphic magnification, so a range of spectral resolving powers can be used without affecting the way slitlet images at the image slicer map to the IFS science detector. Nevertheless, the beam angle through the grating is different for the $R=5,000$ and $R=10,000$ gratings, so compensating prisms must be used. 
The $R \sim 5,000$ gratings are intended to be the work-horse gratings for GMTIFS. There spectral resolving power is sufficient to work between strong airglow emission lines and is matched to the expected kinematics of many science targets. The 4096-pixel IFS science detector offers the ability to measure more than one photometric pass band in one exposure. This is used efficiently for the $m Z J$ grating, which combines both the $Z$ and $J$ pass bands. The $m J H$ grating will allow the important [Fe II] $1.257 \mu \mathrm{m}$ and [Fe II] $1.644 \mu \mathrm{m}$ emission lines to be measured simultaneously. The $m H K$ grating is centered such that the [Fe II] $1.644 \mu \mathrm{m}$ line can be measured simultaneously with other important diagnostic emission lines in the $K$ band, such as $\mathrm{H}_{2} 1-0 \mathrm{~S}(1) 2.122 \mu \mathrm{m}$ and $\mathrm{H}$ I Br $\gamma 2.166 \mu \mathrm{m}$. This means that the $m H K$ grating does not extend deep into the red end of the $K$ band. [Si VIII] $2.483 \mu \mathrm{m}$ falls at the extreme end of the nominal wavelength range, and is likely to be lost in many slitlets due to the "staircase" pattern of the IFS. Lines of the $\mathrm{H}_{2} 1-0$ Q branch (i.e., $\mathrm{Q}(1)$ at $2.408 \mu \mathrm{m}$ to at least $\mathrm{Q}(5)$ at $2.454 \mu \mathrm{m})$ will be accessible.

The $R=10,000$ gratings are included to provide higher spectral resolving power. They generally record the full wavelength range of one atmospheric window. The broader width of the $K$ band prevents this with a single grating setting, so the $h K$ grating has been offset to the red to allow high-resolution observations of the CO $\Delta \mathrm{v}=2$ absorption bands of stars in galactic nuclei to the highest redshift possible. It should be possible to work further into regions of bad atmospheric transmission with these high spectral resolving power gratings than with the $R=5,000$ gratings.

A fixed-format Three-Mirror Anastigmat (TMA) camera is used to image the dispersed IFU pattern on the IFS science detector. It employs three diamond-turned surfaces. The first is a prolate biconic (non-axisymmetric), the second is a strongly oblate biconic (non-axisymmetric), and the third is a slightly oblate conic (axisymmetric and almost spherical). The camera is large, and the first mirror is particularly so with a span of $480 \times 550 \mathrm{~mm}$. State-of-the-art diamondmachining facilities will be needed for its manufacture.

The IFS science detector is a $4096 \times 4096$ Teledyne HgCdTe HAWAII-4RG-15 device. It is mounted on a focusing stage that allows the detector focus to be tuned to each pass band. This detector was chosen for its large $4096 \times 4096$ pixel format, 1-2.5 $\mu \mathrm{m}$ wavelength response, low dark current, and low read noise performance. Read noise is likely to limit the performance of the IFS at the finest image scales.

\subsection{Imager}

The GMTIFS Imager has a field of view of $20.4 \times 20.4$ arcsec that is well matched to the adaptive-optics-corrected field of the single-conjugate LTAO system. The 5 mas pixel size of the imaging mode Nyquist samples the 10 mas diffraction FWHM at the shortest operating wavelength of $\sim 1 \mu \mathrm{m}$ and over-samples the 22 mas diffraction FWHM at $2.2 \mu \mathrm{m}$ for greater fitting precision. Standard broad-band $J H K$ filters are available, as well as a range of intermediate-band filters that are suitable for obtaining photometric redshifts and measuring coarse stellar population characteristics.

The Imager also includes a facility for imaging the telescope exit pupil and the cold pupil mask to ensure their accurate alignment, and so maximize detected signal and minimize detected background. Accurate centering is achieved by reorienting the GMTIFS cryostat in angle with respect to the telescope optical axis. Accurate angular orientation is achieved by adjusting the tracking offset of the rotary cold stop pupil mask in the Fore-Optics. It is envisaged that this alignment will be performed infrequently.

Achieving high Strehl ratio imaging requires that static non-common-path wave-front errors within GMTIFS be measured and nulled using the adaptive secondary mirror. Two defocus lenses allow the measurement of out-of-focus stellar images that will be used with a curvature-sensing algorithm to measure non-common-path wave-front errors at the Imager detector.

In addition to its scientific role, the Imager also acts as the acquisition camera for the IFS.

The optical beam is directed to the Imager by the Science Selector mechanism at the rear of the Fore-Optics (Figure 5). This is the transmitted beam of either the IFS dichroic, or an anti-reflection-coated clear optic for Imager-only science observations.

The GMTIFS Imager design is based on the strong heritage of the Gemini South Adaptive Optics Imager ${ }^{4}$ (GSAOI) that was built by the ANU and is in use with the CANOPUS Multi-Conjugate Adaptive Optics system on the Gemini South telescope. The Imager uses refractive optics to form a pseudo-collimated beam in which the Imager filters are placed. Refractive optics then reimage the sky onto the Imager science detector at an image scale of $5 \mathrm{mas} / \mathrm{pixel}$, which is suitable for diffraction-limited imaging. 
The Imager science detector is also a 4096 $\times 4096$ Teledyne HgCdTe HAWAII-4RG-15 device. It is mounted on a similar focusing stage as will be used for the IFS science detector to allow for different focus positions for each filter. The Imager performance is expected to be limited by sky noise in all broad-band filters, and probably by read noise in the narrow-band filters.

The pupil reimaging lens in the Utility Wheel can be deployed in the Imager optical path to reimage the cold stop pupil onto the Imager science detector. The deployable positive and negative defocus lenses are also located in the Utility Wheel and can be deployed in the Imager beam to measure non-common-path wave-front errors.

\subsection{On-Instrument Wave-Front Sensor Feed}

The GMTIFS OIWFS will access NGSs over the full 180 arcsec diameter guide field passed to GMTIFS from the GMT tertiary mirror, excluding the central $\sim 20.4 \times 20.4$ arcsec science field. The chosen NGS is directed to the OIWFS by the OIWFS feed located within the central optical bench (Figure 9). The OIWFS itself is part of the LTAO system.

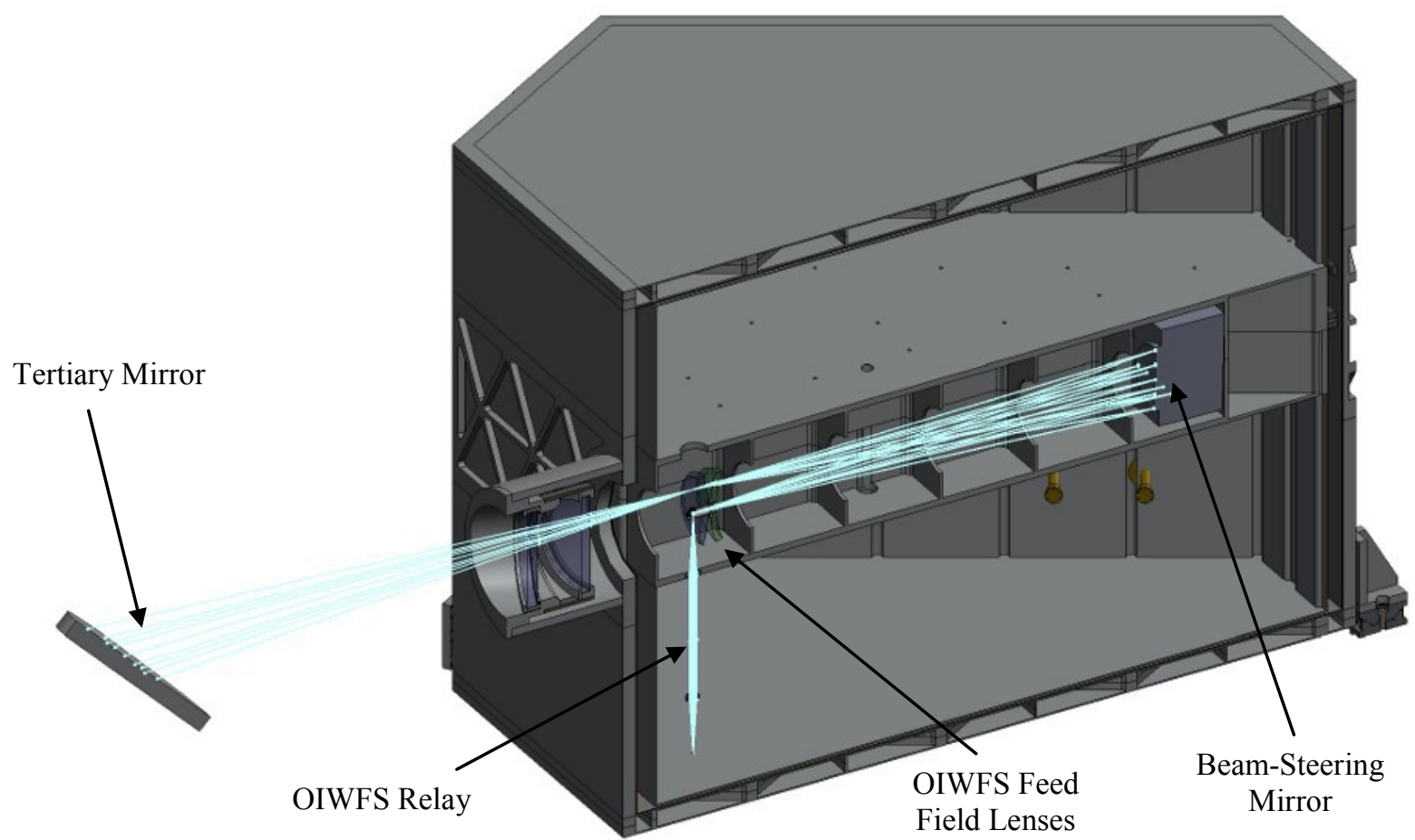

Figure 9: GMTIFS On-Instrument Wave-Front Sensor feed inside the main optical bench.

The near-infrared tip-tilt WFS in the GMTIFS OIWFS acts as the primary position reference for GMTIFS. Both IFS and Imager nodding and dither sequences are executed by mapping out the offset pattern with the OIWFS feed. This ensures that accurate blind offset patterns are executed, even when the science target is too faint to provide accurate postprocessing centroids in individual science exposures. There is therefore a tight requirement on the OIWFS feed in absolute positioning accuracy and repeatability. At the finest IFS scale, we require a RMS positioning accuracy of $\sim 10 \%$ of the diffraction FWHM at the shortest operating wavelength of $0.9 \mu \mathrm{m}$, which corresponds to a RMS positioning accuracy of $\sim 0.9 \mu \mathrm{m}$. A similar requirement exists for GMTIFS Imager observations.

This stringent requirement on the positioning accuracy of the OIWFS feed is expected to be difficult to meet. A mechanical X-Y probe arm at the $\mathrm{f} / 8$ focus could be used as the OIWFS feed. This has the disadvantage that it is the tip of the arm extending into the guide field that must be set to an absolute accuracy of $\sim 1 \mu \mathrm{m}$, so the requirement on the drive mechanism is even more severe. This and other considerations make a mechanical X-Y probe arm unattractive. An alternative that appears to be feasible is to use a beam-steering mirror located at a pupil image to select NGSs by tilting 
this mirror. Translations at the edge of the tilt mirror are significantly smaller than $1 \mu \mathrm{m}$, but may be accommodated with piezo-electric actuators. The baseline OIWFS feed concept employs such a system (Figure 9 and Figure 10).

A two-element field lens (Figure 4 and Figure 9) is located near the $180 \mathrm{~mm}$ diameter input focal plane to form a pupil image on the beam-steering mirror. This mirror has a concave spherical surface with its center of curvature on the telescope focal plane, and so forms a return image of the guide star on that plane. The mirror is tipped and tilted about its vertex to steer this image through a hole in the field lenses, where it is intercepted by the OIWFS pick-off mirror on the back of the science field pick-off mirror, and directed to the OIWFS through a field relay (Figure 9). A weak meniscus corrector lens is placed between the field lens and the beam-steering mirror (Figure 1). This allows good aberration control of the field image without introducing anamorphic distortion in the pupil image.

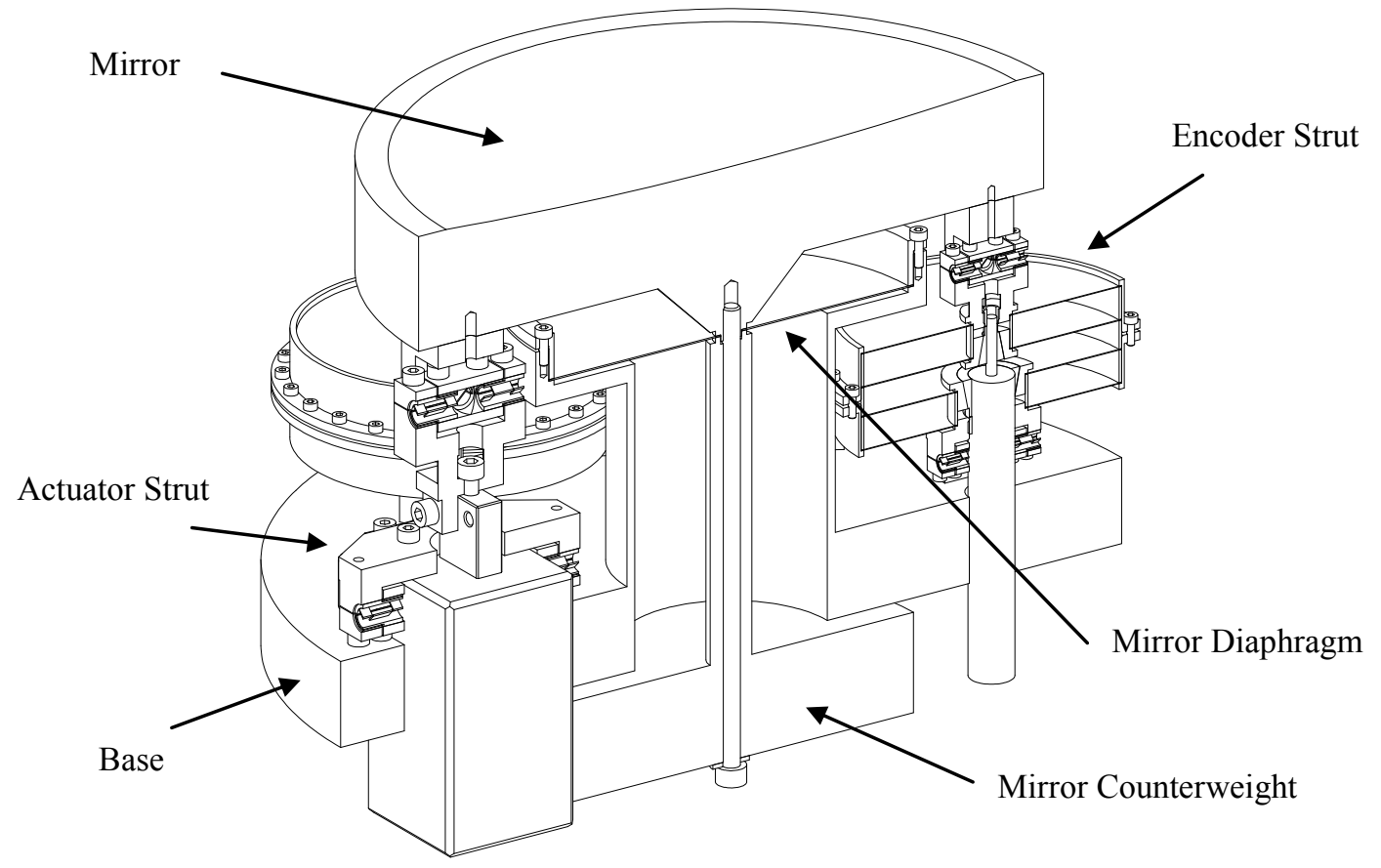

Figure 10: Cutaway view of the GMTIFS Beam-Steering Mirror assembly.

For an on-axis guide star, the imagery would be concentric and therefore free of aberration from the beam-steering mirror. The tip-tilt of the beam-steering mirror that is required to capture the off-axis guide star generates astigmatism that is proportional to the square of the decenter. This is corrected by incorporating a fourth-order aspheric figure into one surface of each field lens, so that they act as a Gascoigne corrector. Applying the aspheric to both lenses gives achromatic correction. The corrector lens also includes a fourth-order aspheric figure. The whole system also corrects the astigmatism of the aplanatic telescope optics.

The tip-tilt range of the beam-steering mirror required to patrol the $180 \mathrm{~mm}$ diameter guide field is 3.5 degrees. The required guiding accuracy and repeatability is $1 / 180,000$ of this range. This corresponds to 1 mas in angle on the sky, and $1 \mu \mathrm{m}$ of image movement in the telescope focal plane. Control of angular mirror movements must then be at a level of 70 mas. To achieve this accuracy, the beam-steering mirror must consolidate fine resolution with low hysteresis. A pointing model will be used to account for flexure between the mirror and the OIWFS, and for non-linearity in the mirror-position sensors. Hysteresis is minimized by avoiding sliding and rolling joints in favor of elastic flexure joints in both the actuator and encoder systems, and the mirror supports.

The beam-steering mirror must move in tip, tilt, and axial translation, but be rigid in the three remaining degrees of freedom. This is achieved using a swash-plate mechanism consisting of three actuators and corresponding feedback loops (Figure 10). The mirror has a diameter of $240 \mathrm{~mm}$ and an edge thickness of $40 \mathrm{~mm}$, while the base has a diameter 
of $300 \mathrm{~mm}$ and a thickness of $30 \mathrm{~mm}$. The mirror is radially supported by means of a flexible diaphragm that also restrains the mirror against axial rotation. Under radial load, the mirror is balanced about the diaphragm by a counterweight. The suspended mass should be slightly counterweight-heavy such that lateral flexure causes the mirror to pivot about its center of curvature.

Axial support is provided by three equally-spaced piezoelectric actuator struts. In Figure 10, tip-tilt position is sensed by three linear-variable-differential-transformer (LVDT) encoder struts, although alternative sensing technologies are being explored. The actuator and encoder struts are equally placed about a pitch circle diameter of $200 \mathrm{~mm}$. The required strut resolution is therefore $17 \mathrm{~nm}$, and the strut position must be repeatable to this level.

The actuator struts are articulated by means of Hooke's joints at each end to accommodate the changing alignment that occurs with tip-tilt. The piezo-electric element is a Physik Instrumente (PI) N-216 Nexline linear actuator with a rated stroke of $20 \mathrm{~mm}$. Communications with PI indicate that the N-216 actuator is capable of being modified for cryogenic operation, but performance may be reduced. Whilst the N-216 is available with in-built linear scale encoders, these do not function at cryogenic temperatures. The PI N-216 actuator achieves a velocity of $0.4 \mathrm{~mm} / \mathrm{s}$ in micro-step mode (at room temperature), so it can traverse the full translation range of $\sim 6 \mathrm{~mm}$ in 15 seconds.

The LVDT (LIN52 LVDT from RDP Group) transformer sleeve and armature core are clamped by collets to opposite sides of a diaphragm flexure capsule (Figure 10). This capsule accurately guides the core within the sleeve, keeping them coaxial. In this sense, mechanical contact between the two components is avoided, and the movement is frictionless.

\section{SUMMARY}

GMTIFS will be an exciting new capability that will significantly extend studies of the physics of individual astronomical objects beyond what is possible with existing and planned instrumentation on 8-10 m telescopes. It will provide a capability that will place the GMT community on par with communities having access to similar instruments on the Thirty Meter Telescope and the European Extremely Large Telescope. And, it will provide opportunities in several areas for synergistic studies with JWST and ALMA.

The design is technically challenging in a number of areas. These risks are being resolved through prototyping activities during the current Preliminary Design phase.

\section{ACKNOWLEDGEMENTS}

Australian membership in the GMT consortium is funded by the Department of Innovation, Industry, Science and Research, and Tertiary Education (DIISRTE) through the Australian Government Education Investment Fund (EIF). The GMTIFS Conceptual Design Study was funded by EIF, the Giant Magellan Telescope Organization (GMTO), and the National Science Foundation under Science Program No. 10 as issued for support of the Giant Segmented Mirror Telescope for the United States Astronomical Community in accordance with Proposal No. AST-0443999 submitted by AURA.

\section{REFERENCES}

[1] Hart, J., McGregor, P. J., \& Bloxham, G. J., "NIFS Concentric Integral-Field Unit”, Proc. SPIE, 4841, 319-329 (2003).

[2] McGregor, P. J., Hart, J., Conroy, P. G., Pfitzner, M. L., Bloxham, G. J., Jones, D. J., Downing, M. D., Dawson, M., Young, P., Jarnyk, M., \& van Harmelen, J., "Gemini Near-infrared Integral-Field Spectrograph (NIFS)", Proc. SPIE, 4841, 1581-1591 (2003).

[3] Dopita, M., Hart, J., McGregor, P., Oates, P., Bloxham, G., \& Jones, D., “The Wide Field Spectrograph (WiFeS)", Ap\&SS, 310, 255-268 (2007).

[4] McGregor, P. J., Hart, J., Stevanovic, D., Bloxham, G., Jones, D., van Harmelen, J., Griesbach, J., Dawson, M., Young, P., \& Jarnyk, M., "Gemini South Adaptive Optics Imager (GSAOI)”, Proc. SPIE, 5492, 1033-1044 (2004). 